\title{
Phytopharmazie
}

\section{Weißdorn bremst die Gefäßalterung}

_ Arterielle Gefäßerkrankungen hängen eng mit den Alterungsprozessen des Endothels zusammen. Im Laufe der Zeit produziert diese Zellschicht immer weniger gefäßschützende Substanzen wie das essenzielle Stickstoffmonoxid (NO). Gleichzeitig werden immer mehr aggressive Sauerstoffverbindungen freigesetzt - es entsteht oxidativer Stress. Eine dadurch ausgelöste komplexe Kettenreaktion stört die empfindliche biochemische Balance in der Gefäßwand, das lokale Angiotensin-System wird hochreguliert. Am Ende des Prozesses stehen verengte und verhärtete Gefäße. Ein typischer Seneszenz-Marker in Endothelzellen ist die erhöhte Aktivität des Enzyms $\beta$-Galactosidase.

Der Weißdornspezialextrakt WS ${ }^{\circ}$ 1442 kann diese Vorgänge bremsen. In einer Untersuchung an Endothelzellen zeigte sich, dass der Extrakt (Wirkstoff in Crataegutt ${ }^{\circ}$ ) die alterungsbedingten Beeinträchtigungen der endothelialen Stickstoffmonoxidsynthase bremst und zur Produktion von ausreichenden Mengen von gefäßschützendem NO führt.

\section{Symptome der Herzinsuffizienz werden gelindert}

Zudem verminderte der Extrakt oxidativen Stress, die Hochregulation des lokalen Angiotensin-Systems und die Aktivität der seneszenzassoziierten $\beta$-Galactosidase. Auf diese Weise könnte das pflanzliche Arzneimittel bei beginnender Herzinsuffizienz die Symptome und die körperliche Belastbarkeit bessern.

Red.

- Khemais-Benkhiat S et al. J Gerontol A Biol Sci Med Sci, online 15. Dezember 2015

\section{Kurz notiert}

Zulassungserweiterung für Daclatasvir $\rightarrow$ Die Europäische Kommission hat für Daclatasvir $\left(\right.$ Daklinza $\left.{ }^{\oplus}\right)$ jetzt Behandlungsregime zur Therapie der chronischen Infektion mit dem Hepatitis-C-Virus (HCV) für drei neue Patientenpopulationen genehmigt. Diese Erweiterung der empfohlenen Behandlungsregime für Daclatasvir in Kombination mit Sofosbuvir (mit oder ohne Ribavirin, abhängig von Indikation und HCV-Genotyp) betrifft HCV-Patienten mit dekompensierter Zirrhose, mit einer HIV-1-Koinfektion sowie einer HCV-Reinfektion nach Lebertransplantation. Sie gilt für alle 28 Mitgliedsstaaten der Europäischen Union sowie Norwegen, Island und Liechtenstein.

Daclatasvir ist kontraindiziert in Kombination mit Arzneimitteln, die starke Induktoren für CYP3A4 und P-Glycoprotein-Transporter sind, da dies zu einer geringeren Exposition und einem Wirksamkeitsverlust von Daclatasvir führen kann. Daclatasvir darf nicht als Monotherapie angewendet werden.

Red.

- Nach Informationen von Bristol-Myers Squibb

\section{Kopfschmerzen vom Spannungstyp}

\section{Pfefferminzöllösung überzeugt in Beobachtungsstudie}

_ Die Wirksamkeit und Verträglichkeit der 10\%igen Pfefferminzöllösung (Prüfpräparat: Euminz ${ }^{\circ}$ bei Spannungskopfschmerzen wurde erneut in einer neuen, nicht-interventionellen Studie bestätigt.

\section{Nach dem ersten Tag kopfschmerzfrei} An der Studie nahmen 271 Patienten mit leichten bis mittelschweren Kopfschmerzen vom Spannungstyp teil. Das speziell entwickelte medizinische Konzentrat aus der Pfefferminze (Menthae piperitae aetheroleum) wurde mithilfe eines Dosier-Pads großflächig auf Stirn und Schläfen aufgetragen.

Weit mehr als $80 \%$ der Teilnehmer beurteilten die Wirkung der medizinisch aufbereiteten Pfefferminze als sehr gut oder gut. Bereits am ersten Tag verschwanden die Kopfschmerzen bei 43\% der Studienteilnehmer komplett. Fast die Hälfte der Studienteilnehmer beurteilten die Wirksamkeit der Öllösung als vergleichbar stark wie die häufig zur Kopfschmerztherapie eingesetzten Präparate Acetylsalicylsäure oder Paracetamol.

\section{Topisches Pfefferminzöl jetzt auch in der Leitlinie}

Das Arzneimittel eignet sich insbesondere aufgrund der guten Verträglichkeit auch für Kinder ab sechs Jahren, für Personen mit empfindlichem Magen und für Menschen, die Schwierigkeiten haben, Tabletten zu schlucken. Das Pfefferminzöl Euminz ${ }^{\oplus}$ ist als einziges lokal anzuwendendes, pflanzliches Arzneimittel zur Behandlung von leichten bis mittelschweren Kopfschmerzen vom Spannungstyp zugelassen.

Die Wirkung ist der Einnahme von Paracetamol 1.000 mg oder Acetylsalicylsäure $1.000 \mathrm{mg}$ ebenbürtig, so die Leitlinie der Deutschen Gesellschaft für

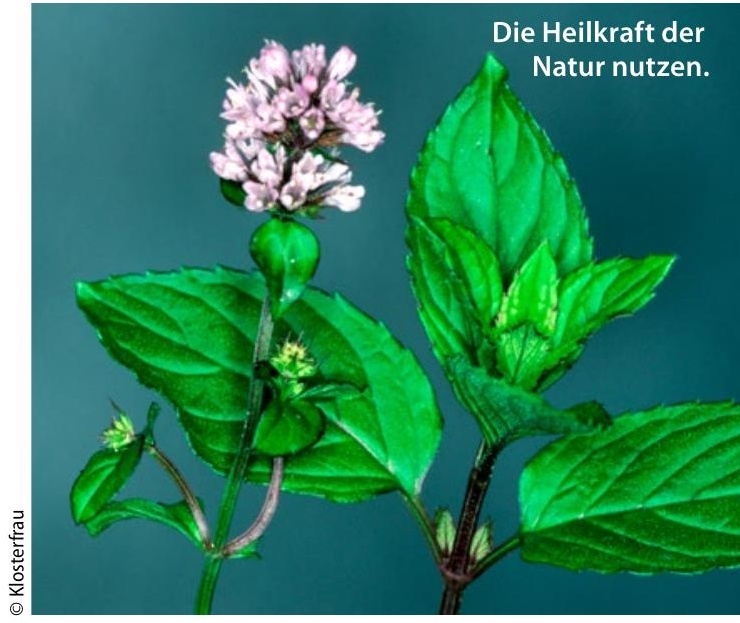

Schmerzmedizin. Für sechs- bis zwölfjährige Kinder ist das Arzneimittel verordnungs- und erstattungsfähig.

Red.

- Nach Informationen von Klosterfrau 